\title{
Fitted numerical scheme for solving singularly perturbed parabolic delay partial differential equations
}

\author{
Mesfin Mekuria Woldaregay and Gemechis File Duressa
}

\begin{abstract}
In this paper, exponentially fitted finite difference scheme is developed for solving singularly perturbed parabolic delay partial differential equations having small delay on the spatial variable. The term with the delay is approximated using Taylor series approximation. The resulting singularly perturbed parabolic partial differential equation is treated using implicit Euler method in the temporal discretization with exponentially fitted operator finite difference method in the spatial discretization. The parameter uniform convergence analysis has been carried out with the order of convergence one. Test examples and numerical results are considered to validate the theoretical analysis of the scheme.
\end{abstract}

Keywords. Delay differential equations, exponentially fitted scheme, uniform convergence

\section{Introduction}

In Mathematical biology, there are different models, in which small values of diffusive parameter play a significant role to capture the behavior of the physical phenomena. Small values of the diffusive parameter in the differential equations have been seen in different physiological process. For example in hemoglobin molecules and oxygen in blood have diffusion coefficient of order $10^{-7}$ and $10^{-5} \mathrm{~cm}^{2} / \mathrm{s}$, respectively $[11,12]$.

The feasibility of recording single neuron movement induces the development of accurate mathematical models of neuronal variability[3]. The modeling of spiking movement of neuron to any level of exactness, one has to consider special features of each kind of neuron and its input processes. In 1965, Stein [19] developed a mathematical model for the stochastic movement of neuron. After two years, the author gives a generalized model to handle a distribution of past synaptic potential amplitudes [20].

In 1991, Musila and Lansky [13] gives a generalizetion on the Stein's model and proposed the mathematical model to consider the time evolution trajectories of the membrane potential in the form of singularly perturbed parabolic delay partial differential equations (SPPDPDEs)

$$
\frac{\partial u}{\partial t}=\frac{\sigma^{2}}{2} \frac{\partial^{2} u}{\partial x^{2}}+\left(\mu_{D}-\frac{x}{\tau}\right) \frac{\partial u}{\partial x}+\lambda_{s} u\left(x+a_{s}, t\right)+\omega_{s} u\left(x+i_{s}, t\right)-\left(\lambda_{s}+\omega_{s}\right) u(x, t),
$$

Received date: March 4, 2020; Published online: March 22, 2021. 2010 Mathematics Subject Classification. 65M06, 65M12, 65M15. Corresponding author: Gemechis File Duressa. 
where the first derivative term is due to the exponential decay between two consecutive jumps caused by the input processes. The membrane potential decays exponentially to the resting level with a membrane time constant $\tau . \mu_{D}$ and $\sigma$ are diffusion moments of Wiener process characterizing the influence of dendritic synapses on the cell excitability. The excitatory input contributes to the membrane potential by an amplitude $a_{s}$ with intensity $\lambda_{s}$ and similarly the inhibitory input contributes by an amplitude $i_{s}$ with intensity $\omega_{s}$.

The model in (1.1) is in the form of SPPDPDEs, which is difficult to drive its exact solution. Thus, to find solution of this model, we have to deal with the numerical techniques. Still know only a few numerical schemes have been developed for solving this type of model problems. In recent years, different scholar's have devoted for the study of numerical solution of SPPDPDEs with the delay on the spatial variable. In papers $[1,2,3]$, Bansal and Sharma have developed different numerical schemes for the problem with general shift arguments (where the delays are large). In [8], Kumar and Kadalbajoo used implicit Euler in temporal direction and B-spline collocation method on piece-wise uniform mesh in spatial direction. In [15], Ramesh and Kadalbajoo used implicit Euler in temporal direction and upwind and midpoint upwind finite difference method on piecewise uniform mesh in spatial direction. Daba and Duressa [5] used implicit Euler method in temporal direction and extended cubic B-spline basis functions consisting of a free parameter for the resulting system of ordinary differential equations in the spatial direction. Shivhare et al. [18] semi-discretized using the Crank Nicolson method in the time direction on uniform mesh and used quadratic B-spline collocation method in the space direction on exponentially graded mesh. In [22, 23] Woldaregay and Duressa applied numerical schemes by using non-standard finite difference scheme for the spatial discretization with Runge Kutta on the temporal direction.

The main contribution of this paper is to develop an exponentially fitted numerical scheme which converges independent of the influence of the perturbation parameter. In addition, to analyse the parameter uniform convergence of the scheme.

Organization of the paper: In section 2, description of the problem and the properties of the analytical solution is given. In section 3 , the discretization and techniques of exponentially fitted finite difference and the parameter uniform convergence of the discrete scheme is given. In section 4, numerical examples and results are given and finally in section 5 , the conclusion of the work is given.

Notations: Throughout the paper $N$ and $M$ are denoted for the number of mesh in space and time discretization. The symbol $C$ is denoted for positive constant independent of $c_{\varepsilon}, N$ and $\Delta t$. The norm $\|\cdot\|$ denotes the supremum norm which is defined as $\|g\|=\max |g(x, t)|, \forall(x, t) \in D$ and in discrete case $\|g\|=\max _{x_{i}, t_{j}}\left|g\left(x_{i}, t_{j}\right)\right|$.

\section{Problem Formulation}

The time dependent singularly perturbed parabolic delay differential equations with delay on the spatial variable of the reaction term is given by

$$
\frac{\partial u}{\partial t}-\varepsilon^{2} \frac{\partial^{2} u}{\partial x^{2}}+a(x) \frac{\partial u}{\partial x}+\alpha(x) u(x-\delta, t)+\beta(x) u(x, t)=f(x, t), \quad(x, t) \in D,
$$

where $D=\Omega \times \Lambda=(0,1) \times(0, T]$ with smooth boundary $\partial D=\bar{D}-D=D_{L} \cup D_{0} \cup D_{R}$ for some positive number $\mathrm{T}$, subject to the initial and interval-boundary conditions

$$
\begin{aligned}
& u(x, 0)=u_{0}(x), x \in D_{0}=\{(x, 0): x \in \bar{\Omega}\}, \\
& u(x, t)=\phi(x, t),(x, t) \in D_{L}=\{(x, t):-\delta \leq x \leq 0, t \in \Lambda\}, \\
& u(1, t)=\psi(1, t),(x, t) \in D_{R}=\{(x, t): x=1, t \in \Lambda\},
\end{aligned}
$$


where, $\varepsilon$ is a singular perturbation parameter satisfying $0<\varepsilon \ll 1$ and $\delta$ is delay parameter assumed to be sufficiently small as order of $o(\varepsilon)$. The coefficient functions $a(x), \alpha(x), \beta(x)$ source function $f(x, t)$ and the initial and boundary solutions $u_{0}(x), \phi(x, t), \psi(1, t)$ are assumed to be sufficiently smooth, bounded and independent of $\varepsilon$. The coefficient functions of reaction term $\alpha(x)$ and $\beta(x)$ are assumed to satisfy the condition $\alpha(x)+\beta(x) \geq \zeta>0, \forall x \in \bar{\Omega}$ where $\zeta$ is constant. This condition ensures the solution of the problem in (2.1)-(2.2) exhibits boundary layer in the neighborhood of $D_{L}$ or $D_{R}$ depending on whether $a(x)-\delta \beta(x)<0$ or $>0$ for $x \in \bar{\Omega}$.

For the case $\delta=o(\varepsilon)$, it is applicable to use a Taylor series approximation for the delay term [21]. In the next subsection, we use this procedure to tackle the delay term. In general, computing numerical solution of singularly perturbed problems by using classical numerical methods leads to oscillations or divergence in computed solutions [15]. To overcome this problem, a large number of mesh points are required when $\varepsilon$ is very small. This is not applicable and fails to give accurate results due to round off error. So, in this paper we develop a uniformly convergent numerical method using implicit Euler in the temporal discretization and exponentially fitted operator finite difference method in the spatial discretization, which treat the problem without creating an oscillations.

\subsection{Approximation for the delay term}

From the assumption $\delta=o(\varepsilon)$, so using Taylor series approximation, we obtain

$$
u(x-\delta, t) \approx u(x, t)-\delta \frac{\partial u}{d x}+\frac{\delta^{2}}{2} \frac{\partial^{2} u}{d x^{2}}+O\left(\delta^{3}\right) .
$$

Using (2.3) into (2.1) and truncating $O\left(\delta^{3}\right)$, we obtain

$$
\frac{\partial u}{\partial t}-c_{\varepsilon}(x) \frac{\partial^{2} u}{\partial x^{2}}+p(x) \frac{\partial u}{\partial x}+q(x) u(x, t)=f(x, t),(x, t) \in D,
$$

where $c_{\varepsilon}(x)=\varepsilon^{2}-\frac{\delta^{2}}{2} \alpha(x), p(x)=a(x)-\delta \alpha(x), q(x)=\alpha(x)+\beta(x)$ with initial and boundary conditions

$$
\begin{aligned}
& u(x, 0)=u_{0}(x), x \in \bar{\Omega}, \\
& u(0, t)=\phi(0, t), t \in \bar{\Lambda}, \\
& u(1, t)=\psi(1, t), t \in \bar{\Lambda} .
\end{aligned}
$$

For small $\delta$, the problems in (2.1)-(2.2) and (2.4)-(2.5) are asymptotically equivalent, since the truncated term is $O\left(\delta^{3}\right)$. By assuming $0<c_{\varepsilon}(x) \leq \varepsilon^{2}-\delta^{2} M_{1}=c_{\varepsilon}$ where $\alpha(x) \geq 2 M_{1}, \forall x \in \Omega$. We assume also $p(x)=a(x)-\delta \alpha(x) \geq p^{*}>0, \forall x \in \Omega$ which imply the existence of the right boundary layer of thickness $O\left(c_{\varepsilon}\right)$. For the case $p(x)=a(x)-\delta \alpha(x) \leq p^{*}<0, \forall x \in \Omega$ imply the existence of left boundary layer of thickness $O\left(c_{\varepsilon}\right)$ and can be treated similarly as the right layer.

\subsection{Properties of the Analytical Solution}

We establish the existence and uniqueness of the solution of (2.4)-(2.5) by the assumption that the data are Holder continuous and imposing appropriate compatibility conditions at the points $(0,0),(1,0)$. 
Lemma 2.1. Let $u_{0}(x) \in C^{2}[0,1], \phi \in C^{1}[0, T]$ and $\psi \in C^{1}[0, T]$ by imposing the compatibility conditions $u_{0}(0)=\phi(0,0), u_{0}(1)=\psi(1,0)$ and

$$
\begin{aligned}
& \frac{\partial \phi(0,0)}{\partial t}-c_{\varepsilon} \frac{\partial^{2} u_{0}(0)}{\partial x^{2}}+p(0) \frac{\partial u_{0}(0)}{\partial x}+q(0) u_{0}(0)=f(0,0), \\
& \frac{\partial \psi(1,0)}{\partial t}-c_{\varepsilon} \frac{\partial^{2} u_{0}(1)}{\partial x^{2}}+p(1) \frac{\partial u_{0}(1)}{\partial x}+q(1) u_{0}(1)=f(1,0),
\end{aligned}
$$

so that the data matches at the two corners $(0,0)$ and $(1,0)$. Let $p(x), q(x)$ and $f(x, t)$ be continuous on $D$. Then the problem (2.4)-(2.5) has unique solution $u \in C^{2}((0,1) \times(0, T])$.

In case of the compatibility conditions are not satisfied, a unique solution may exist but may not be differentiable on all of $\partial D$.

In case $p(x) \geq p^{*}>0$ and $q(x) \geq \zeta>0$, regular boundary layer exist near $x=1$. With the help of the compatibility conditions, we conclude that there exist a constant $C$ independent of $c_{\varepsilon}$ such that $\forall(x, t) \in \bar{D}$, we have the following conditions

$$
\begin{aligned}
|u(x, t)-u(x, 0)| & =\left|u(x, t)-u_{0}(x)\right| \leq C t \text { and } \\
|u(x, t)-u(0, t)| & =|u(x, t)-\phi(0, t)| \leq C(1-x),
\end{aligned}
$$

for the detail one can refer [17],[24].

Remark 1. Since the boundary layer exist on the right side of the domain we have to note that there does not exist $C$ independent of $c_{\varepsilon}$ such that $|u(x, t)-u(1, t)|=|u(x, t)-\psi(1, t)| \leq C x$.

To show the bounds of the solutions $u(x, t)$ of $(2.4)$, we assume, without loss of generality the initial condition to be zero. Since $u_{0}(x)$ is sufficiently smooth and using the property of norm, we prove the following lemma.

Lemma 2.2. The bound on the solution $u(x, t)$ of the problem in (2.4)-(2.5) is given by

$$
|u(x, t)| \leq C, \forall(x, t) \in \bar{D} .
$$

Proof. See on [22].

Let $L$ be a differential operator denoted for the differential equation $L u(x, t)=\frac{\partial u}{\partial t}-c_{\varepsilon} \frac{\partial^{2} u}{\partial x^{2}}+$ $p(x) \frac{\partial u}{\partial x}+q(x) u(x, t)$.

Lemma 2.3. Continuous maximum principle. Let $z$ be a sufficiently smooth function defined on $D$ which satisfies $z(x, t) \geq 0,(x, t) \in \partial D$ and $L z(x, t) \geq 0,(x, t) \in D$. Then, implies that $z(x, t) \geq 0, \forall(x, t) \in \bar{D}$.

Proof. Let $\left(x^{*}, t^{*}\right)$ be such that $z\left(x^{*}, t^{*}\right)=\min _{(x, t) \in \bar{D}} z(x, t)$ and suppose that $z\left(x^{*}, t^{*}\right)<0$. It is clear that $\left(x^{*}, t^{*}\right) \notin \partial D$. From the theory in extrema values in Calculus, since $z\left(x^{*}, t^{*}\right)=$ $\min _{(x, t) \in \bar{D}} z(x, t)$ which implies $z_{x}\left(x^{*}, t^{*}\right)=0, z_{t}\left(x^{*}, t^{*}\right)=0$ and $z_{x x}\left(x^{*}, t^{*}\right) \geq 0$ and implies that $L z\left(x^{*}, t^{*}\right)<0,(x, t) \in D$ which is contradiction to the assumption that made above $L z\left(x^{*}, t^{*}\right) \geq$ $0,(x, t) \in D$. Hence, $z(x, t) \geq 0, \forall(x, t) \in \bar{D}$.

Lemma 2.4. Uniform Stability Estimate. Let $u(x, t)$ be the solution of the problem in (2.4)(2.5). Then, we obtain the bound

$$
|u(x, t)| \leq \zeta^{-1}\|f\|+\max \left\{\left|u_{0}(x)\right|,|\phi(0, t)|,|\psi(1, t)|\right\} .
$$


Proof. We define two barrier functions $\vartheta^{ \pm}(x, t)$ as $\vartheta^{ \pm}(x, t)=\zeta^{-1}\|f\|+\max \left\{\left|u_{0}(x)\right|,|\phi(0, t)|,|\psi(1, t)|\right\} \pm u(x, t)$. At the initial value, we have

$$
\vartheta^{ \pm}(x, 0)=\zeta^{-1}\|f\|+\max \left\{\left|u_{0}(x)\right|,|\phi(0, t)|,|\psi(1, t)|\right\} \pm u(x, 0) \geq 0 .
$$

On the boundaries, we have

$$
\begin{aligned}
& \vartheta^{ \pm}(0, t)=\zeta^{-1}\|f\|+\max \left\{\left|u_{0}(x)\right|,|\phi(0, t)|,|\psi(1, t)|\right\} \pm u(0, t) \geq 0, \\
& \vartheta^{ \pm}(1, t)=\zeta^{-1}\|f\|+\max \left\{\left|u_{0}(x)\right|,|\phi(0, t)|,|\psi(1, t)|\right\} \pm u(1, t) \geq 0,
\end{aligned}
$$

and for the differential operator

$$
\begin{aligned}
L \vartheta^{ \pm}(x, t)= & \vartheta_{t}^{ \pm}(x, t)-c_{\varepsilon} \vartheta_{x x}^{ \pm}(x, t)+p(x) \vartheta_{x}^{ \pm}(x, t)+q(x) \vartheta^{ \pm}(x, t) \\
= & \left(0 \pm u_{t}(x, t)\right)-c_{\varepsilon}\left(0 \pm u_{x x}(x, t)\right)+p(x)\left(0 \pm u_{x}(x, t)\right) \\
& +q(x)\left(\zeta^{-1}\|f\|+\max \left\{\left|u_{0}(x)\right|,|\phi(0, t)|,|\psi(1, t)|\right\} \pm u(x, t)\right) \\
= & q(x)\left(\zeta^{-1}\|f\|+\max \left\{\left|u_{0}(x)\right|,|\phi(0, t)|,|\psi(1, t)|\right\}\right) \pm f(x, t) \\
\geq & 0, \text { since } q(x) \geq \zeta>0,
\end{aligned}
$$

which implies $L \vartheta^{ \pm}(x, t) \geq 0$. Hence by using maximum principle, we obtain $\vartheta^{ \pm}(x, t) \geq 0, \forall(x, t) \in$ $\bar{D}$.

Lemma 2.5. The bounds for the derivative of the solution $u(x, t)$ of the problem in (2.4)-(2.5) are given by

$$
\left|\frac{\partial^{k+l} u(x, t)}{\partial x^{k} \partial t^{l}}\right| \leq C\left(1+c_{\varepsilon}^{-k} e^{-p^{*}(1-x) / c_{\varepsilon}}\right), 0 \leq k \leq 4,0 \leq k+l \leq 4 .
$$

Proof. For the proof one can refer [4].

\section{Numerical Scheme Formulation}

\subsection{Temporal Discretization}

Let sub-divide the temporal domain $[0, T]$ into $\mathrm{M}$ sub-intervals as $t_{0}=0, t_{j}=j \Delta t, j=$ $0,1,2, \ldots, M$, where $\Delta t=T / M$. In the first step, we semi-discretize the problem (2.4)-(2.5) using the implicit Euler method. In this stage of discretization, a system of BVPs are obtained as

$$
\frac{U^{j+1}(x)-U^{j}(x)}{\Delta t}-c_{\varepsilon} \frac{d^{2}}{d x^{2}} U^{j+1}(x)+p(x) \frac{d}{d x} U^{j+1}(x)+q(x) U^{j+1}(x)=f\left(x, t_{j+1}\right),
$$

where $U^{j+1}(x)$ is denoted for the approximation of $u\left(x, t_{j+1}\right)$ at the $(j+1)$ th time level discretization. Rearranging we write as

$$
L^{\Delta t} U^{j+1}(x)=g\left(x, t_{j+1}\right), x \in \bar{\Omega}, j=0,1,2, \ldots, M-1,
$$

with discretized boundary conditions

$$
U^{j+1}(0)=\phi\left(0, t_{j+1}\right), \quad U^{j+1}(1)=\psi\left(1, t_{j+1}\right),
$$


where

$$
L^{\Delta t} U^{j+1}(x)=-c_{\varepsilon} \frac{d^{2}}{d x^{2}} U^{j+1}(x)+p(x) \frac{d}{d x} U^{j+1}(x)+d(x) U^{j+1}(x)
$$

and

$$
g\left(x, t_{j+1}\right)=\frac{1}{\Delta t} U^{j}(x)+f\left(x, t_{j+1}\right)
$$

for $d(x)=\frac{1}{\Delta t}+q(x)$. Here $d(x) \geq \zeta^{*}>0$, since $q(x)>0$.

Lemma 3.1. Semi-discrete maximum principle. Let $z^{j+1}$ be a sufficiently smooth function on the domain $\bar{\Omega}$. If $z^{j+1}(0) \geq 0, z^{j+1}(1) \geq 0$ and $L^{\Delta t} z^{j+1}(x) \geq 0, x \in \Omega$, then $z^{j+1}(x) \geq$ $0, \forall x \in \bar{\Omega}$.

Proof. Let $x^{*}$ be such that $z^{j+1}\left(x^{*}\right)=\min _{x \in \bar{\Omega}} z^{j+1}(x)$ and suppose that $z^{j+1}\left(x^{*}\right)<0$. From the above assumption it is clear that $x^{*} \notin\{0,1\}$. Which implies that $x^{*} \in(0,1)$. Applying the property in calculus, we have $\left(z^{j+1}\right)_{x x}\left(x^{*}\right) \geq 0$ and $\left(z^{j+1}\right)_{x}\left(x^{*}\right)=0$. So we obtain $L^{\Delta t} z^{j+1}\left(x^{*}\right)<$ 0 which is contradiction to $L^{\Delta t} z^{j+1}\left(x^{*}\right) \geq 0, x \in \bar{\Omega}$. Therefore we conclude that $z^{j+1}(x) \geq$ $0, \forall x \in \bar{\Omega}$.

Next, we analyze the discretization error. Let us denote $u\left(x, t_{j+1}\right)$ and $U^{j+1}(x)$ be the exact and approximate solution of the problem in (2.4)-(2.5) using the above discretization. Let us denote the local error by $e^{j+1}(x):=u\left(x, t_{j+1}\right)-U^{j+1}(x), j=0,1,2, \ldots, M-1$

Lemma 3.2. Local error estimate. Suppose that $\left|\frac{\partial^{l}}{\partial t^{l}} u(x, t)\right| \leq C, \quad(x, t) \in \bar{\Omega} \times \Lambda, \quad 0 \leq l \leq 2$. The local error estimate in the temporal direction is given by

$$
\left\|e_{j+1}\right\| \leq C_{1}(\Delta t)^{2} .
$$

Proof. Using the series expansion to $u\left(x, t_{j+1}\right)$, we obtain

$$
u\left(x, t_{j+1}\right)=u\left(x, t_{j}\right)+\Delta t u_{t}\left(x, t_{j}\right)+O\left((\Delta t)^{2}\right) .
$$

Substituting (3.5) into (2.4), we obtain

$$
\begin{aligned}
\frac{u\left(x, t_{j+1}\right)-u\left(x, t_{j}\right)}{\Delta t}= & u_{t}\left(x, t_{j}\right)+O\left((\Delta t)^{2}\right) \\
= & -\left(-c_{\varepsilon} u\left(x, t_{j}\right)_{x x}+p(x) u\left(x, t_{j}\right)_{x}+q(x) u\left(x, t_{j}\right)-f\left(x+t_{j}\right)\right) \\
& +O\left((\Delta t)^{2}\right) .
\end{aligned}
$$

Since error satisfies the differential equations. So the local error satisfies the semi-discrete operator

$$
\begin{aligned}
L^{\Delta t} e^{j+1} & =O\left((\Delta t)^{2}\right), \\
e^{j+1}(0) & =0=e^{j+1}(1) .
\end{aligned}
$$

Hence, applying the maximum principle, we obtain

$$
\left\|e^{j+1}\right\| \leq C_{1}(\Delta t)^{2} .
$$

Next, we need to give the bound for the global error of the semi-discretization. Let denote $T E^{j+1}$ be the global error estimate up to the $(j+1) t h$ time step. 
Lemma 3.3. Global error estimate. The global error estimate at $t_{j+1}$ is given by

$$
\left\|T E^{j+1}\right\| \leq C(\Delta t), j=0,1,2, \ldots, M-1 .
$$

Proof. Using the results in local error estimate up to the $(j+1)$ th time step in Lemma 3.2, the global error estimate is obtained as

$$
\begin{aligned}
\left\|T E^{j+1}\right\| & =\left\|\sum_{l=1}^{j+1} e^{l}\right\| \\
& \leq\left\|e^{1}\right\|+\left\|e^{2}\right\|+\ldots+\left\|e^{j+1}\right\| \\
& \leq C_{1}(j \Delta t)(\Delta t) \\
& \leq C_{1} T(\Delta t), \quad \text { since }(j+1) \Delta t \leq T \\
& =C(\Delta t), \quad C_{1} T=C,
\end{aligned}
$$

where $C$ is constant independent of $c_{\varepsilon}$ and $\Delta t$.

In the above section, the continuous problem is semi-discretized and converted to a system of BVPs given in (3.2)-(3.3). Next, we set a bound for the derivatives of solution of the problems in (3.2)-(3.3).

Lemma 3.4. For each $j=0,1,2, \ldots, M-1$. The derivative of solution of the boundary value problems in (3.2)-(3.3) satisfies the bound

$$
\left|\frac{d^{k} U^{j+1}(x)}{d x^{k}}\right| \leq C\left(1+c_{\varepsilon}^{-k} e^{-\frac{p^{*}(1-x)}{c_{\varepsilon}}}\right), \quad x \in \bar{\Omega}, 0 \leq k \leq 4 .
$$

Proof. Direct from Lemma 2.5.

\subsection{Spatial Discretization}

Now, the spatial domain $[0,1]$ is discretized into $N$ equal number of subintervals, each of length $h=1 / N$. Let $x_{0}=0, x_{N}=1$ and $x_{i}=i h, i=1,2, \ldots, N-1$. To find the numerical solution of system of syngularly perturbed BVPs, we use exponentially fitted operator finite difference method (FOFDM). In the considered case the boundary layer is on the right side of the domain i.e. near $x=1$. From the theory of singular perturbations given in O'Malley [14] for singularly perturbed boundary value problem of the form in (3.2)-(3.3), the asymptotic solution up to zero order approximation is given as:

$$
U^{j+1}(x)=U_{0}^{j+1}(x)+\left(\psi\left(1, t_{j+1}\right)-U_{0}^{j+1}(1)\right) \exp \left(-\int_{x}^{1}\left(\frac{p(x)}{c_{\varepsilon}}-\frac{d(x)}{p(x)}\right) d x\right) .
$$

Using Taylor's series expansion for $p(x)$ about $x=1$ and restriction to their first terms and simplifying, we get the asymptotic solution as

$$
U^{j+1}(x)=U_{0}^{j+1}(x)+\left(\psi\left(1, t_{j+1}\right)-U_{0}^{j+1}(1)\right) \exp \left(-\frac{p(1)(1-x)}{c_{\varepsilon}}\right),
$$

where $U_{0}^{j+1}$ is the solution of the reduced problems (obtained by setting $c_{\varepsilon}=0$ ) given by

$$
p(x) \frac{d}{d x} U_{0}^{j+1}(x)+d(x) U_{0}^{j+1}(x)=g^{j+1}(x), \quad U_{0}^{j+1}(0)=\phi\left(0, t_{j+1}\right) .
$$


Considering $h$ is small enough, the discretized form of (3.11) becomes

$$
U^{j+1}(i h)=U_{0}^{j+1}(i h)+\left(\psi\left(1, t_{j+1}\right)-U_{0}^{j+1}(1)\right) \exp \left(-p(1)\left(1 / c_{\varepsilon}-i \rho\right)\right)
$$

Using Taylor series we approximate $U_{0}^{j+1}((i \pm 1) h)$ by $U_{0}^{j+1}(i h)$ and we obtain

$$
\begin{aligned}
& U^{j+1}((i-1) h)=U_{0}^{j+1}(i h)+\left(\psi\left(1, t_{j+1}\right)-U_{0}^{j+1}(1)\right) \exp \left(-p(1)\left(1 / c_{\varepsilon}-(i-1) \rho\right)\right), \\
& U^{j+1}((i+1) h)=U_{0}^{j+1}(i h)+\left(\psi\left(1, t_{j+1}\right)-U_{0}^{j+1}(1)\right) \exp \left(-p(1)\left(1 / c_{\varepsilon}-(i+1) \rho\right)\right),
\end{aligned}
$$

where $\rho=h / c_{\varepsilon}, \quad h=1 / N$.

To handle the effect of the perturbation parameter artificial viscosity (exponentially fitting factor $\sigma(\rho))$ is multiplied on the term containing the perturbation parameter as

$$
-c_{\varepsilon} \sigma(\rho) \frac{d^{2}}{d x^{2}} U^{j+1}(x)+p(x) \frac{d}{d x} U^{j+1}(x)+d(x) U_{j+1}(x)=g^{j+1}(x) .
$$

Next, considering a uniform grid points $\bar{\Omega}^{N}=\left\{x_{i}\right\}_{i=0}^{N}$ and denoting $h=x_{i+1}-x_{i}$, for any mesh function $z_{i}$, define the forward, backward and central finite difference for first derivative approximation as

$$
D^{+} z_{i}=\frac{z_{i+1}-z_{i}}{h}, \quad D^{-} z_{i}=\frac{z_{i}-z_{i-1}}{h} \text { and } D^{0} z_{i}=\frac{z_{i+1}-z_{i-1}}{2 h}
$$

respectively and the second derivative approximation as

$$
D^{+} D^{-} z_{i}=\frac{z_{i+1}-2 z_{i}+z_{i-1}}{h^{2}} .
$$

Applying the central finite difference scheme on (3.15) takes the form

$$
-c_{\varepsilon} \sigma(\rho) D^{+} D^{-} U^{j+1}\left(x_{i}\right)+p\left(x_{i}\right) D^{0} U^{j+1}\left(x_{i}\right)+d\left(x_{i}\right) U^{j+1}\left(x_{i}\right)=g^{j+1}\left(x_{i}\right) .
$$

Equivalently (3.16) is rewritten as

$$
L^{h, \Delta t} U_{i}^{j+1} \equiv-c_{\varepsilon} \sigma(\rho) \frac{U_{i-1}^{j+1}-2 U_{i}^{j+1}+U_{i+1}^{j+1}}{h^{2}}+p\left(x_{i}\right) \frac{U_{i+1}^{j+1}-U_{i-1}^{j+1}}{2 h}+d\left(x_{i}\right) U_{i}^{j+1}=g_{i}^{j+1} .
$$

Multiplying (3.17) by $h$ and considering $h$ is small and truncating the term $h\left(g_{i}^{j+1}-d\left(x_{i}\right) U_{i}^{j+1}\right)$, (since $g_{i}^{j+1}-d\left(x_{i}\right) U_{i}^{j+1}$ is bounded) results to

$$
-\frac{\sigma(\rho)}{\rho}\left(U_{i-1}^{j+1}-2 U_{i}^{j+1}+U_{i+1}^{j+1}\right)+\frac{p\left(x_{i}\right)}{2}\left(U_{i+1}^{j+1}-U_{i-1}^{j+1}\right)=0
$$

which gives

$$
\sigma(\rho)=\frac{\rho p\left(x_{i}\right)}{2} \frac{U_{i+1}^{j+1}-U_{i-1}^{j+1}}{U_{i-1}^{j+1}-2 U_{i}^{j+1}+U_{i+1}^{j+1}} .
$$

From (3.13) and (3.14) we obtain the difference

$$
\begin{aligned}
U_{i-1}^{j+1}-2 U_{i}^{j+1}+U_{i+1}^{j+1}= & \left(\psi\left(t_{j+1}\right)-U_{0}^{j+1}(1)\right) \exp \left(-p(1)\left(1 / c_{\varepsilon}-i \rho\right) \times\right. \\
& {[\exp (p(1) \rho)-2+\exp (-p(1) \rho)], } \\
U_{i+1}^{j+1}-U_{i-1}^{j+1}= & \left(\psi\left(t_{j+1}\right)-U_{0}^{j+1}(1)\right) \exp \left(-p(1)\left(1 / c_{\varepsilon}-(i) \rho\right)\right) \times \\
& {[\exp (-p(1) \rho)-\exp (p(1) \rho)] . }
\end{aligned}
$$


Now using the results in (3.19) into (3.18) gives

$$
\begin{aligned}
\sigma(\rho) & =\frac{\rho p\left(x_{i}\right)}{2} \frac{\exp (-p(1) \rho)-\exp (p(1) \rho)}{\exp (p(1) \rho)-2+\exp (-p(1) \rho)} \\
& =\frac{\rho p\left(x_{i}\right)}{2} \frac{\left(\exp \left(\frac{-p(1) \rho}{2}\right)-\exp \left(\frac{p(1) \rho}{2}\right)\right)\left(\exp \left(\frac{-p(1) \rho}{2}\right)+\exp \left(\frac{p(1) \rho}{2}\right)\right)}{\left(\exp \left(\frac{p(1) \rho}{2}\right)-\exp \left(\frac{-p(1) \rho}{2}\right)\right)^{2}},
\end{aligned}
$$

simplifying the exponential fitting factor is obtained as

$$
\sigma(\rho)=\frac{\rho p\left(x_{i}\right)}{2} \operatorname{coth}\left(\frac{\rho p(1)}{2}\right) .
$$

Hence, the required finite difference scheme becomes

$L^{h, \Delta t} U_{i}^{j+1} \equiv\left(\frac{c_{\varepsilon} \sigma(\rho)}{h^{2}}-\frac{p\left(x_{i}\right)}{2 h}\right) U_{i-1}^{j+1}+\left(\frac{-2 c_{\varepsilon} \sigma(\rho)}{h^{2}}+d\left(x_{i}\right)\right) U_{i}^{j+1}+\left(\frac{c_{\varepsilon} \sigma(\rho)}{h^{2}}+\frac{p\left(x_{i}\right)}{2 h}\right) U_{i+1}^{j+1}=g_{i}^{j+1}$

for $i=1,2, \ldots, N-1$ and $j=0,1,2, \ldots, M-1$.

\subsection{Stability and Uniform Convergence Analysis}

Next, we need to prove the discrete maximum principle for the scheme in (3.17).

Lemma 3.5. Discrete maximum principle. Assume that the mesh function $z^{j+1}\left(x_{i}\right)$ satisfies $z^{j+1}\left(x_{0}\right) \geq 0$ and $z^{j+1}\left(x_{N}\right) \geq 0$. If $L^{h, \Delta t} z^{j+1}\left(x_{i}\right) \geq 0$ for $1 \leq i \leq N-1$, then $z^{j+1}\left(x_{i}\right) \geq 0$ $\forall i, 0 \leq i \leq N$.

Proof. Let choose $k$ such that $z^{j+1}\left(x_{k}\right)=\min _{x_{i}} z^{j+1}\left(x_{i}\right), 1 \leq i \leq N-1$. If $z^{j+1}\left(x_{k}\right) \geq 0$, the the proof completed. We can see that $z^{j+1}\left(x_{k+1}\right)-z^{j+1}\left(x_{k}\right) \geq 0$ and $z^{j+1}\left(x_{k}\right)-z^{j+1}\left(x_{k-1}\right) \leq 0$. Now from (3.17), we obtain $L^{h, \Delta t} z^{j+1}\left(x_{k}\right)<0$ which contradicts $L^{h, \Delta t} z^{j+1}\left(x_{k}\right)<0$. Hence, the assumption is wrong. We conclude that $z^{j+1}\left(x_{i}\right) \geq 0, \forall i, 0 \leq i \leq N$.

Lemma 3.6. Uniform stability estimate. The solution $U_{i}^{j+1}$ of the discrete scheme in (3.17) satisfy the bound

$$
\left|U_{i}^{j+1}\right| \leq \frac{\max \left|L^{h, \Delta t} U_{i}^{j+1}\right|}{\zeta^{*}}+\max \left\{\left|\phi\left(0, t_{j+1}\right)\right|,\left|\psi\left(1, t_{j+1}\right)\right|\right\}
$$

Proof. Let $p=\frac{\max \left|L^{h, \Delta t} U_{i}^{j+1}\right|}{\zeta^{*}}+\max \left\{\left|\phi\left(0, t_{j+1}\right)\right|,\left|\psi\left(1, t_{j+1}\right)\right|\right\}$ and define the barrier function $\vartheta_{i, j+1}^{ \pm}$by $\vartheta_{i, j+1}^{ \pm}=p \pm U_{i}^{j+1}$. On the boundary points, we obtain

$$
\begin{gathered}
\vartheta_{0, j+1}^{ \pm}=p \pm U_{0}^{j+1}=\frac{\max \left|L^{h, \Delta t} U_{i}^{j+1}\right|}{\zeta^{*}}+\max \left\{\left|\phi\left(0, t_{j+1}\right)\right|,\left|\psi\left(1, t_{j+1}\right)\right|\right\} \pm \phi\left(0, t_{j+1}\right) \geq 0 . \\
\vartheta_{N, j+1}^{ \pm}=p \pm U_{N}^{j+1}=\frac{\max \left|L^{h, \Delta t} U_{i}^{j+1}\right|}{\zeta^{*}}+\max \left\{\left|\phi\left(0, t_{j+1}\right)\right|,\left|\psi\left(1, t_{j+1}\right)\right|\right\} \pm \psi\left(1, t_{j+1}\right) \geq 0 .
\end{gathered}
$$


On the discretized spatial domain $x_{i}, 1<i<N-1$, we obtain

$$
\begin{aligned}
L^{h, \Delta t} \vartheta_{i, j+1}^{ \pm} & =-c_{\varepsilon} \sigma(\rho)\left(\frac{p \pm U_{i+1}^{j+1}-2\left(p \pm U_{i}^{j+1}\right)+p \pm U_{i-1}^{j+1}}{h^{2}}\right) \\
& +p\left(x_{i}\right)\left(\frac{p \pm U_{i+1}^{j+1}-p \pm U_{i-1}^{j+1}}{2 h}\right)+d\left(x_{i}\right)\left(p \pm U_{i}^{j+1}\right) \\
& =d\left(x_{i}\right) p \pm L^{h, \Delta t} U_{i}^{j+1} \\
& =d\left(x_{i}\right)\left(\frac{\max \left|L^{h, \Delta t} U_{i}^{j+1}\right|}{\zeta^{*}}+\max \left\{\left|\phi\left(0, t_{j+1}\right)\right|,\left|\psi\left(1, t_{j+1}\right)\right|\right\}\right) \pm g^{j+1}\left(x_{i}\right) \\
& \geq 0, \quad \text { since } d\left(x_{i}\right) \geq \zeta^{*} .
\end{aligned}
$$

From Lemma 3.5, we obtain $\vartheta_{i, j+1}^{ \pm} \geq 0, \forall x_{i} \in \bar{\Omega}^{N}$. Hence the required bound is obtained.

The following theorem gives the truncation error of the developed scheme.

Theorem 3.1. Let $U^{j+1}\left(x_{i}\right)$ and $U_{i}^{j+1}$ be respectively the continuous solution of (3.2)-(3.3) and approximate(discrete) solutions of (3.21). Then, for sufficiently large $N$, the following truncation error estimate holds:

$$
\left|L^{h, \Delta t}\left(U^{j+1}\left(x_{i}\right)-U_{i}^{j+1}\right)\right| \leq \frac{C N^{-2}}{N^{-1}+c_{\varepsilon}}\left(1+c_{\varepsilon}^{-3} \exp \left(-\frac{p^{*}\left(1-x_{i}\right)}{c_{\varepsilon}}\right)\right) .
$$

Proof. Let us consider the local truncation error in space discretization as

$$
\begin{aligned}
\left|L^{h, \Delta t}\left(U^{j+1}\left(x_{i}\right)-U_{i}^{j+1}\right)\right|= & \left|-c_{\varepsilon} \sigma(\rho)\left(\frac{d^{2}}{d x^{2}}-D^{+} D^{-}\right) U^{j+1}\left(x_{i}\right)+p\left(x_{i}\right)\left(\frac{d}{d x}-D^{0}\right) U^{j+1}\left(x_{i}\right)\right| \\
\leq & \left|-c_{\varepsilon}\left[p\left(x_{i}\right) \frac{\rho}{2} \operatorname{coth}\left(p(1) \frac{\rho}{2}\right)-1\right] D^{+} D^{-} U^{j+1}\left(x_{i}\right)\right| \\
& +\left|c_{\varepsilon}\left(\frac{d^{2}}{d x^{2}}-D^{+} D^{-}\right) U^{j+1}\left(x_{i}\right)\right|+\left|p\left(x_{i}\right)\left(\frac{d}{d x}-D^{0}\right) U^{j+1}\left(x_{i}\right)\right|,
\end{aligned}
$$

where $\sigma(\rho)=p\left(x_{i}\right) \frac{\rho}{2} \operatorname{coth}\left(p(1) \frac{\rho}{2}\right)$, and $\rho=\frac{N^{-1}}{c_{\varepsilon}}$.

Let $C_{1}$ and $C_{2}$ are constants we have $\rho \operatorname{coth}(\rho)-1 \leq C_{1} \rho^{2}$ for $\rho \leq 1$. For $\rho \rightarrow \infty$, since $\lim _{\rho \rightarrow \infty} \operatorname{coth}(\rho)=1$ gives $\rho \operatorname{coth}(\rho)-1 \leq C_{1} \rho$. In general for all $\rho>0$ we have

$$
\begin{gathered}
C_{1} \frac{\rho^{2}}{\rho+1} \leq \rho \operatorname{coth}(\rho)-1 \leq C_{2} \frac{\rho^{2}}{\rho+1} \text { and } \\
c_{\varepsilon}[\rho \operatorname{coth}(\rho)-1] \leq c_{\varepsilon} \frac{\left(N^{-1} / c_{\varepsilon}\right)^{2}}{N^{-1} / c_{\varepsilon}+1}=\frac{N^{-2}}{N^{-1}+c_{\varepsilon}} \text { since, } \rho=N^{-1} / c_{\varepsilon} .
\end{gathered}
$$

From Taylor series expansion we obtain the bound as

$$
\begin{gathered}
\left|D^{+} D^{-} U^{j+1}\left(x_{i}\right)\right| \leq C\left|\frac{d^{2} U^{j+1}\left(x_{i}\right)}{d x^{2}}\right|, \\
\left|\left(\frac{d^{2}}{d x^{2}}-D^{+} D^{-}\right) U^{j+1}\left(x_{i}\right)\right| \leq C N^{-2}\left|\frac{d^{4} U^{j+1}\left(x_{i}\right)}{d x^{4}}\right|,
\end{gathered}
$$

Similarly for first derivative term,

$$
\left|\left(\frac{d}{d x}-D^{0}\right) U^{j+1}\left(x_{i}\right)\right| \leq C N^{-2}\left|\frac{d^{3} U^{j+1}\left(x_{i}\right)}{d x^{3}}\right| .
$$


Using the bounds for the differences of the derivatives in (3.24),(3.25) and (3.26) into (3.3) gives

$$
\begin{aligned}
\left|L^{h, \Delta t}\left(U^{j+1}\left(x_{i}\right)-U_{i, j+1}\right)\right| \leq & C \frac{N^{-2}}{N^{-1}+c_{\varepsilon}}\left|\frac{d^{2} U^{j+1}\left(x_{i}\right)}{d x^{2}}\right|+c_{\varepsilon} C N^{-2}\left|\frac{d^{4} U^{j+1}\left(x_{i}\right)}{d x^{4}}\right| \\
& +C N^{-2}\left|\frac{d^{3} U^{j+1}\left(x_{i}\right)}{d x^{3}}\right| \\
\leq & \frac{N^{-2}}{N^{-1}+c_{\varepsilon}}\left|\frac{d^{2} U^{j+1}\left(x_{i}\right)}{d x^{2}}\right|+C N^{-2}\left[c_{\varepsilon}\left|\frac{d^{4} U^{j+1}\left(x_{i}\right)}{d x^{4}}\right|\right. \\
& \left.+\left|\frac{d^{3} U^{j+1}\left(x_{i}\right)}{d x^{3}}\right|\right] .
\end{aligned}
$$

Here, the target is to show the scheme convergence independent of the perturbation parameter. Using the bounds for the derivatives of the solution in Lemma 3.4, we obtain

$$
\begin{aligned}
\left|L^{h, \Delta t}\left(U^{j+1}\left(x_{i}\right)-U_{i}^{j+1}\right)\right| \leq & \frac{C N^{-2}}{N^{-1}+c_{\varepsilon}}\left(1+c_{\varepsilon}^{-2} \exp \left(-\frac{p^{*}\left(1-x_{i}\right)}{c_{\varepsilon}}\right)\right) \\
& +C N^{-2}\left[c_{\varepsilon}\left(1+c_{\varepsilon}^{-4} \exp \left(-\frac{p^{*}\left(1-x_{i}\right)}{c_{\varepsilon}}\right)\right)\right. \\
& \left.+\left(1+c_{\varepsilon}^{-3} \exp \left(-\frac{p^{*}\left(1-x_{i}\right)}{c_{\varepsilon}}\right)\right)\right] \\
\leq & \frac{C N^{-2}}{N^{-1}+c_{\varepsilon}}\left(1+c_{\varepsilon}^{-2} \exp \left(-\frac{p^{*}\left(1-x_{i}\right)}{c_{\varepsilon}}\right)\right) \\
& +C N^{-2}\left[\left(c_{\varepsilon}+c_{\varepsilon}^{-3} \exp \left(-\frac{p^{*}\left(1-x_{i}\right)}{c_{\varepsilon}}\right)\right)\right. \\
& \left.+\left(1+c_{\varepsilon}^{-3} \exp \left(-\frac{p^{*}\left(1-x_{i}\right)}{c_{\varepsilon}}\right)\right)\right] .
\end{aligned}
$$

Since $c_{\varepsilon}^{-3} \geq c_{\varepsilon}^{-2}$, we obtain

$$
\left|L^{h, \Delta t}\left(U^{j+1}\left(x_{i}\right)-U_{i}^{j+1}\right)\right| \leq \frac{C N^{-2}}{N^{-1}+c_{\varepsilon}}\left(1+c_{\varepsilon}^{-3} \exp \left(-\frac{p^{*}\left(1-x_{i}\right)}{c_{\varepsilon}}\right)\right),
$$

which gives the required bound.

Lemma 3.7. For a fixed number of mesh numbers $N$ and for $c_{\varepsilon} \rightarrow 0$, it holds

$$
\lim _{c_{\varepsilon} \rightarrow 0} \max _{i} \frac{\exp \left(-\frac{p^{*}\left(1-x_{i}\right)}{c_{\varepsilon}}\right)}{c_{\varepsilon}^{m}}=0, \quad m=1,2,3, \ldots
$$

where $x_{i}=i h, h=1 / N, \forall i=1,2, \cdots, N-1$.

Proof. Interested reader can refer from [23].

Using Lemma 3.7 into (3.27) results to

$$
\left|L^{h, \Delta t}\left(U^{j+1}\left(x_{i}\right)-U_{i}^{j+1}\right)\right| \leq \frac{C N^{-2}}{N^{-1}+c_{\varepsilon}} .
$$

Hence, by the the discrete maximum principle we obtain

$$
\left|U^{j+1}\left(x_{i}\right)-U_{i}^{j+1}\right| \leq \frac{C N^{-2}}{N^{-1}+c_{\varepsilon}} .
$$


Remark 2. From the bound in (3.30) one can observe that for the case $c_{\varepsilon}>N^{-1}$ the scheme secures second order convergence. We expect to lose an order of convergence for small values of $c_{\varepsilon}$, and in fact it turns out that the scheme is first-order uniformly convergent.

Theorem 3.2. Let $u$ and $U$ be respectively the exact solution of (2.4)-(2.5) and numerical solutions of (3.21) on discretized domain. Then for sufficiently large $N$, the following parameter uniform error estimate holds:

$$
\sup _{0<c_{\varepsilon} \leq 1}\|u-U\| \leq C\left(N^{-1}+\Delta t\right) .
$$

Proof. Immediate result from (3.8) and (3.30).

\section{Numerical Examples and Discussion}

To validate the theoretical justifications and results in this paper, we developed an algorithm and perform experiments using the proposed scheme for the problem in (2.1)-(2.2).

Example 1. From [22] consider the problem

$$
\frac{\partial u}{\partial t}-\varepsilon^{2} \frac{\partial^{2} u}{\partial x^{2}}+\left(2+x+x^{2}\right) \frac{\partial u}{\partial x}+\left(\frac{1+x^{2}}{2}\right) u(x-\delta, t)=\sin (\pi x(1-x))
$$

with initial interval-boundary conditions $u_{0}(x)=0$, on $x \in[0,1], \phi(x, t)=0$, on $x \in[-\delta, 0]$, $\psi(x, t)=0, x=1$ and $T=1$.

Example 2. From [22] consider the problem

$$
\frac{\partial u}{\partial t}-\varepsilon^{2} \frac{\partial^{2} u}{\partial x^{2}}+\frac{\partial u}{\partial x}+\left(2-x^{2}\right) u(x-\delta, t)+\left(1+x^{2}+\exp (x)\right) u(x, t)=50(x(1-x))^{3}
$$

with initial interval-boundary conditions $u_{0}(x)=0$, on $x \in[0,1], \phi(x, t)=0$, on $x \in[-\delta, 0]$, $\psi(x, t)=0, x=1$ and $T=2$.

Exact solution of the examples given above are not known. With the help of double mesh techniques, we compute the maximum point-wise absolute error, $\varepsilon$-uniform error, rate of convergence and $\varepsilon$-uniform rate of convergence of the scheme by using the double mesh techniques. We define the maximum point-wise absolute error as

$$
E_{\varepsilon, \delta}^{N, M}=\max _{i, j}\left|U_{i, j}^{N, M}-U_{i, j}^{2 N, 2 M}\right|,
$$

where $N, M$ are the number of mesh points in spatial and temporal direction respectively. Let $U_{i, j}^{N, M}$ be the computed solution of (2.4)-(2.5) using $N, M$ mesh points and let $U_{i, j}^{2 N, 2 M}$ be the computed solution on double number of mesh points $2 N, 2 M$ by including the mid points $x_{i+1 / 2}=$ $\frac{x_{i+1}+x_{i}}{2}$ and $t_{j+1 / 2}=\frac{t_{j+1}+t_{j}}{2}$ into the mesh points.

Next, we calculate the $\varepsilon$-uniform error estimate by using the formula

$$
E^{N, M}=\max _{\varepsilon, \delta}\left(E_{\varepsilon, \delta}^{N, M}\right) .
$$

We calculate the rate of convergence of the scheme by using the formula

$$
r_{\varepsilon, \delta}^{N, M}=\log _{2}\left(\frac{E_{\varepsilon, \delta}^{N, M}}{E_{\varepsilon, \delta}^{2 N, 2 M}}\right) .
$$


and similarly we calculate the $\varepsilon$ - uniform rate of convergence by using the formula

$$
r^{N, M}=\log _{2}\left(\frac{E^{N, M}}{E^{2 N, 2 M}}\right) .
$$

The solutions of the above two examples exhibits a right boundary layer of thickness $O\left(c_{\varepsilon}\right)$ near $x=1$. One can clearly observe from the Figures 1 and 2 the formation of the boundary layer as $\varepsilon$ goes small. In Figure 3 the effect of the delay parameter on the solution is show. When the magnitude of delay grows the thickness of the boundary layer decreases as see on the Figure 3 (a) and (b). From Figure 4 on the Log-Log scale plot, one can see that while the perturbation parameter goes very small the scheme converges uniformly independent of the perturbation parameter. This is the main result claimed to shown in this paper. In Table 1 and 3 one can observe the maximum absolute error of the scheme. In these tables, in each column as the perturbation parameter goes small the maximum absolute error becomes stable and uniform. From the results in Tables 1-4 the developed scheme is more accurate than the results in [15], [16] and [22] and in addition that it is parameter uniform convergent with order of convergence one.

Table 1: Example 1, maximum absolute error of the proposed scheme for $\delta=0.6 \varepsilon$.

\begin{tabular}{lcccccc}
\hline$\varepsilon$ & $\mathrm{N}=32$ & 64 & 128 & 256 & 512 & 1024 \\
$\downarrow$ & $\mathrm{M}=60$ & 120 & 240 & 480 & 960 & 1920 \\
\hline $2^{0}$ & $1.7851 \mathrm{e}-05$ & $4.3116 \mathrm{e}-06$ & $9.9398 \mathrm{e}-07$ & $2.0898 \mathrm{e}-07$ & $5.8327 \mathrm{e}-08$ & $3.3946 \mathrm{e}-08$ \\
$2^{-2}$ & $1.2040 \mathrm{e}-03$ & $3.3664 \mathrm{e}-04$ & $8.6686 \mathrm{e}-05$ & $2.1835 \mathrm{e}-05$ & $5.4690 \mathrm{e}-06$ & $1.3679 \mathrm{e}-06$ \\
$2^{-4}$ & $2.7316 \mathrm{e}-03$ & $1.5408 \mathrm{e}-03$ & $7.9725 \mathrm{e}-04$ & $3.3395 \mathrm{e}-04$ & $1.0489 \mathrm{e}-04$ & $2.8136 \mathrm{e}-05$ \\
$2^{-6}$ & $2.7035 \mathrm{e}-03$ & $1.5252 \mathrm{e}-03$ & $8.1049 \mathrm{e}-04$ & $4.1778 \mathrm{e}-04$ & $2.1210 \mathrm{e}-04$ & $1.0683 \mathrm{e}-04$ \\
$2^{-8}$ & $2.6965 \mathrm{e}-03$ & $1.5213 \mathrm{e}-03$ & $8.0835 \mathrm{e}-04$ & $4.1667 \mathrm{e}-04$ & $2.1153 \mathrm{e}-04$ & $1.0657 \mathrm{e}-04$ \\
$2^{-10}$ & $2.6948 \mathrm{e}-03$ & $1.5203 \mathrm{e}-03$ & $8.0781 \mathrm{e}-04$ & $4.1640 \mathrm{e}-04$ & $2.1139 \mathrm{e}-04$ & $1.0650 \mathrm{e}-04$ \\
$2^{-12}$ & $2.6944 \mathrm{e}-03$ & $1.5200 \mathrm{e}-03$ & $8.0768 \mathrm{e}-04$ & $4.1633 \mathrm{e}-04$ & $2.1136 \mathrm{e}-04$ & $1.0649 \mathrm{e}-04$ \\
$2^{-14}$ & $2.6943 \mathrm{e}-03$ & $1.5200 \mathrm{e}-03$ & $8.0765 \mathrm{e}-04$ & $4.1631 \mathrm{e}-04$ & $2.1135 \mathrm{e}-04$ & $1.0648 \mathrm{e}-04$ \\
$2^{-16}$ & $2.6942 \mathrm{e}-03$ & $1.5200 \mathrm{e}-03$ & $8.0764 \mathrm{e}-04$ & $4.1631 \mathrm{e}-04$ & $2.1135 \mathrm{e}-04$ & $1.0648 \mathrm{e}-04$ \\
$2^{-18}$ & $2.6942 \mathrm{e}-03$ & $1.5200 \mathrm{e}-03$ & $8.0764 \mathrm{e}-04$ & $4.1631 \mathrm{e}-04$ & $2.1135 \mathrm{e}-04$ & $1.0648 \mathrm{e}-04$ \\
$2^{-20}$ & $2.6942 \mathrm{e}-03$ & $1.5200 \mathrm{e}-03$ & $8.0764 \mathrm{e}-04$ & $4.1631 \mathrm{e}-04$ & $2.1135 \mathrm{e}-04$ & $1.0648 \mathrm{e}-04$ \\
& & & & & & \\
$E^{N, M}$ & $2.7316 \mathrm{e}-03$ & $1.5408 \mathrm{e}-03$ & $8.1049 \mathrm{e}-04$ & $4.1778 \mathrm{e}-04$ & $2.1210 \mathrm{e}-04$ & $1.0683 \mathrm{e}-04$ \\
$r^{N, M}$ & 0.8261 & 0.9268 & 0.9561 & 0.9780 & 0.9894 & - \\
\hline
\end{tabular}

\section{Conclusion}

An exponentially fitted numerical method is developed for solving singularly perturbed parabolic delay differential equations with the delay on the spatial variable of the reaction term. We consider the case when the solution exhibits a boundary layer at $\mathrm{x}=1$. First, the considered problem is converted to asymptotically equivalent singularly perturbed parabolic PDEs by applying Taylor's

For interpretation of the references to color in this figure legend, the reader is referred to the web version of this article. 
Table 2: Example 1; $\varepsilon$-uniform error $\left(E^{N . M}\right)$ and $\varepsilon$-uniform rate of convergence $\left(r^{N, M}\right)$ of the proposed method and results in [22], [16] and (in [15] for the case $\delta=0.9 \varepsilon$ ).

\begin{tabular}{lcccccc}
\hline Schemes & $\mathrm{N}=32$ & 64 & 128 & 256 & 512 & 1024 \\
$\downarrow$ & $\mathrm{M}=60$ & 120 & 240 & 480 & 960 & 1920 \\
\hline Proposed & $2.7316 \mathrm{e}-03$ & $1.5408 \mathrm{e}-03$ & $8.1049 \mathrm{e}-04$ & $4.1778 \mathrm{e}-04$ & $2.1210 \mathrm{e}-04$ & $1.0683 \mathrm{e}-04$ \\
Scheme & 0.8261 & 0.9268 & 0.9561 & 0.9780 & 0.9894 & - \\
Result & $3.9288 \mathrm{e}-03$ & $1.9657 \mathrm{e}-03$ & $9.8350 \mathrm{e}-04$ & $4.9194 \mathrm{e}-04$ & $2.4602 \mathrm{e}-04$ & $1.2302 \mathrm{e}-04$ \\
in [22] & 0.9990 & 0.9991 & 0.9994 & 0.9997 & 0.9999 & - \\
Result & $5.9125 \mathrm{e}-03$ & $3.6155 \mathrm{e}-03$ & $2.1167 \mathrm{e}-03$ & $1.1957 \mathrm{e}-03$ & $6.5593 \mathrm{e}-04$ & $3.5160 \mathrm{e}-04$ \\
in [16] & 0.7096 & 0.7724 & 0.8240 & 0.8662 & 0.8996 & 0.9256 \\
Upwind & $8.3467 \mathrm{e}-03$ & $5.3894 \mathrm{e}-03$ & $3.3649 \mathrm{e}-03$ & $2.0082 \mathrm{e}-03$ & $1.1571 \mathrm{e}-03$ & $6.4904 \mathrm{e}-04$ \\
in [15] & 0.6311 & 0.6796 & 0.7447 & 0.7954 & 0.8341 & - \\
Mid pt Up & $8.3467 \mathrm{e}-03$ & $5.3894 \mathrm{e}-03$ & $3.3649 \mathrm{e}-03$ & $2.0082 \mathrm{e}-03$ & $1.1571 \mathrm{e}-03$ & $6.4904 \mathrm{e}-04$ \\
wind in [15] & 0.6311 & 0.6796 & 0.7447 & 0.7954 & 0.8341 & - \\
\hline
\end{tabular}

Table 3: Example 2, maximum absolute errors when $\delta=0.5 \varepsilon$.

\begin{tabular}{lcccccc}
\hline$\varepsilon \downarrow$ & $\mathrm{N}=\mathrm{M}=32$ & 64 & 128 & 256 & 512 & 1024 \\
\hline $2^{-0}$ & $2.5106 \mathrm{e}-05$ & $6.2740 \mathrm{e}-06$ & $1.5689 \mathrm{e}-06$ & $3.9220 \mathrm{e}-07$ & $9.8052 \mathrm{e}-08$ & $2.4524 \mathrm{e}-08$ \\
$2^{-2}$ & $2.4000 \mathrm{e}-04$ & $6.0279 \mathrm{e}-05$ & $1.5099 \mathrm{e}-05$ & $3.7831 \mathrm{e}-06$ & $9.4982 \mathrm{e}-07$ & $2.3948 \mathrm{e}-07$ \\
$2^{-4}$ & $2.1542 \mathrm{e}-03$ & $9.1450 \mathrm{e}-04$ & $2.9008 \mathrm{e}-04$ & $7.8109 \mathrm{e}-05$ & $1.9917 \mathrm{e}-05$ & $5.0043 \mathrm{e}-06$ \\
$2^{-6}$ & $2.1620 \mathrm{e}-03$ & $1.1183 \mathrm{e}-03$ & $5.6972 \mathrm{e}-04$ & $2.8736 \mathrm{e}-04$ & $1.4130 \mathrm{e}-04$ & $5.8760 \mathrm{e}-05$ \\
$2^{-8}$ & $2.1503 \mathrm{e}-03$ & $1.1124 \mathrm{e}-03$ & $5.6674 \mathrm{e}-04$ & $2.8591 \mathrm{e}-04$ & $1.4360 \mathrm{e}-04$ & $7.1963 \mathrm{e}-05$ \\
$2^{-10}$ & $2.1473 \mathrm{e}-03$ & $1.1111 \mathrm{e}-03$ & $5.6599 \mathrm{e}-04$ & $2.8553 \mathrm{e}-04$ & $1.4341 \mathrm{e}-04$ & $7.1867 \mathrm{e}-05$ \\
$2^{-12}$ & $2.1466 \mathrm{e}-03$ & $1.1108 \mathrm{e}-03$ & $5.6580 \mathrm{e}-04$ & $2.8543 \mathrm{e}-04$ & $1.4335 \mathrm{e}-04$ & $7.1843 \mathrm{e}-05$ \\
$2^{-14}$ & $2.1464 \mathrm{e}-03$ & $1.1107 \mathrm{e}-03$ & $5.6575 \mathrm{e}-04$ & $2.8541 \mathrm{e}-04$ & $1.4335 \mathrm{e}-04$ & $7.1837 \mathrm{e}-05$ \\
$2^{-16}$ & $2.1464 \mathrm{e}-03$ & $1.1107 \mathrm{e}-03$ & $5.6574 \mathrm{e}-04$ & $2.8540 \mathrm{e}-04$ & $1.4335 \mathrm{e}-04$ & $7.1836 \mathrm{e}-05$ \\
$2^{-18}$ & $2.1464 \mathrm{e}-03$ & $1.1107 \mathrm{e}-03$ & $5.6575 \mathrm{e}-04$ & $2.8540 \mathrm{e}-04$ & $1.4335 \mathrm{e}-04$ & $7.1836 \mathrm{e}-05$ \\
$2^{-20}$ & $2.1464 \mathrm{e}-03$ & $1.1107 \mathrm{e}-03$ & $5.6575 \mathrm{e}-04$ & $2.8540 \mathrm{e}-04$ & $1.4335 \mathrm{e}-04$ & $7.1836 \mathrm{e}-05$ \\
& & & & & & \\
$E^{N, M}$ & $2.1464 \mathrm{e}-03$ & $1.1107 \mathrm{e}-03$ & $5.6575 \mathrm{e}-04$ & $2.8540 \mathrm{e}-04$ & $1.4335 \mathrm{e}-04$ & $7.1836 \mathrm{e}-05$ \\
$r^{N, M}$ & 0.9504 & 0.9732 & 0.9872 & 0.9934 & 0.9968 & - \\
\hline
\end{tabular}

Table 4: Example 2, $\varepsilon$-uniform error $\left(E^{N . M}\right)$ and $\varepsilon$-uniform rate of convergence $\left(r^{N, M}\right)$ of the proposed method and results in [22].

\begin{tabular}{lcccccc}
\hline$\varepsilon \downarrow$ & $\mathrm{N}=\mathrm{M}=32$ & 64 & 128 & 256 & 512 & 1024 \\
\hline Proposed & $2.1503 \mathrm{e}-03$ & $1.1124 \mathrm{e}-03$ & $5.6674 \mathrm{e}-04$ & $2.8591 \mathrm{e}-04$ & $1.4360 \mathrm{e}-04$ & $7.1963 \mathrm{e}-05$ \\
Scheme & 0.9509 & 0.9729 & 0.9871 & 0.9935 & 0.9967 & - \\
Result & $5.7389 \mathrm{e}-03$ & $2.9180 \mathrm{e}-03$ & $1.4718 \mathrm{e}-03$ & $7.3915 \mathrm{e}-04$ & $3.7036 \mathrm{e}-04$ & $1.8537 \mathrm{e}-04$ \\
in $[22]$ & 0.9758 & 0.9874 & 0.9936 & 0.9969 & 0.9985 & - \\
\hline
\end{tabular}



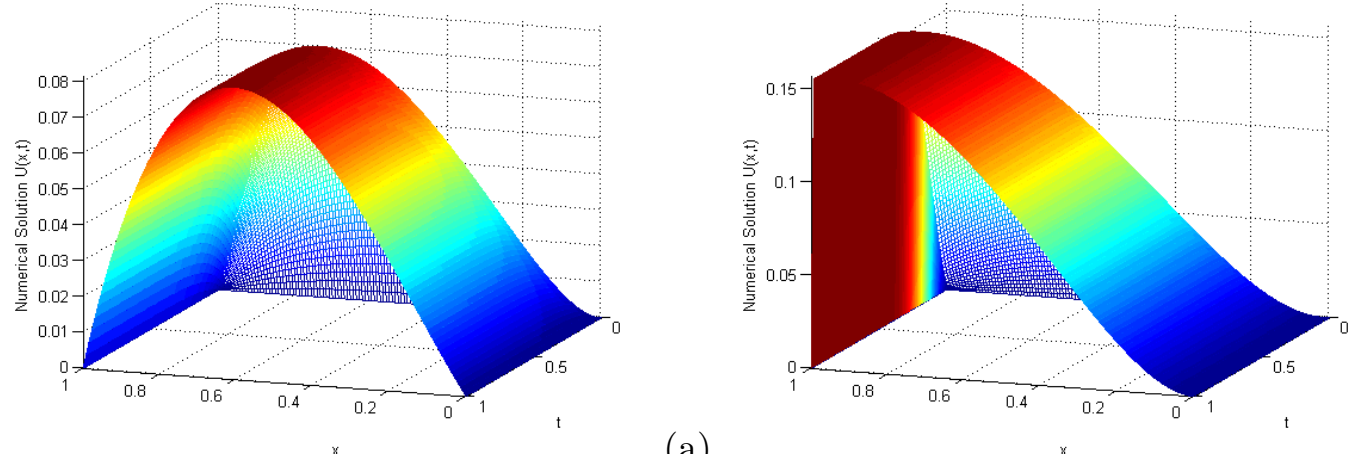

(a)

Figure 1: 3D view of computed solution of Example 1, with the effect of the singular perturbation parameter on (a) for $\varepsilon=2^{0}$, on (b) for $\varepsilon=2^{-20}, \delta=0$. (For interpretation of the references to color in this figure legend, the reader is referred to the web version of this article, DOI:10.5556/j.tkjm.53.2022.3638)
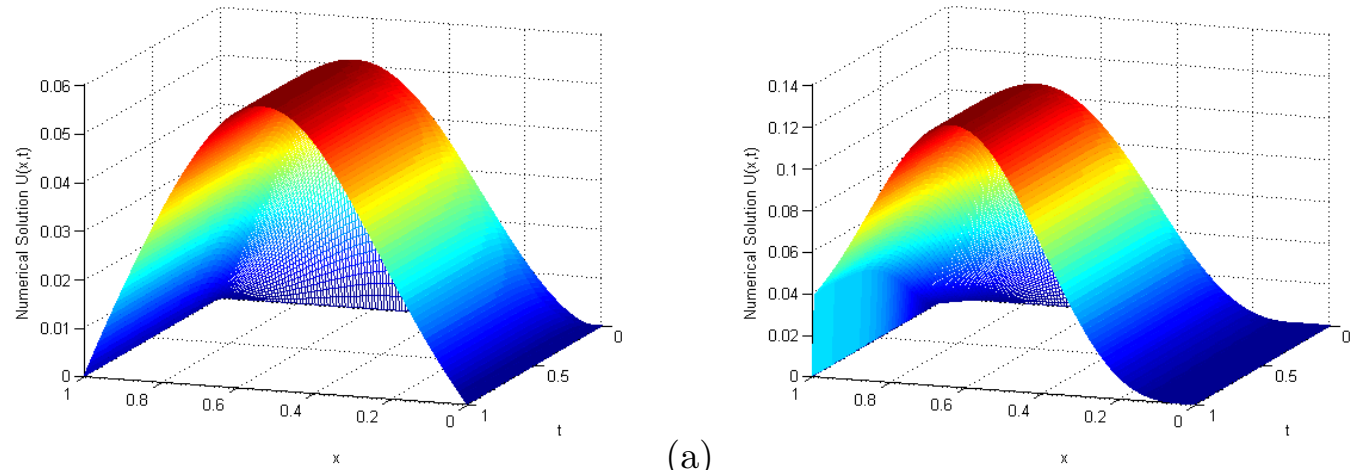

(a)

Figure 2: 3D view of computed solution of Example 2, with the effect of the singular perturbation parameter on (a) for $\varepsilon=2^{0}$, on (b) for $\varepsilon=2^{-20}, \delta=0$. (For interpretation of the references to color in this figure legend, the reader is referred to the web version of this article, DOI:10.5556/j.tkjm.53.2022.3638)

series approximation for the delay term. By inducing exponential fitting factor for terms with perturbation parameters, we developed the discrete scheme. The developed scheme is uniformly convergent (it converges independent of the perturbation parameter). A detail uniform stability and convergence analysis is given to show the uniform convergence of the scheme with rate of convergence $O\left(N^{-1}+\Delta t\right)$. The developed scheme is investigated by considering two test examples. The influence of $\varepsilon$ and $\delta$ on the solution of the problem are shown using figures. The results of the proposed scheme is investigated by comparing the results with published articles. We observed that the proposed method gives more accurate and $\varepsilon$-uniform numerical results. 

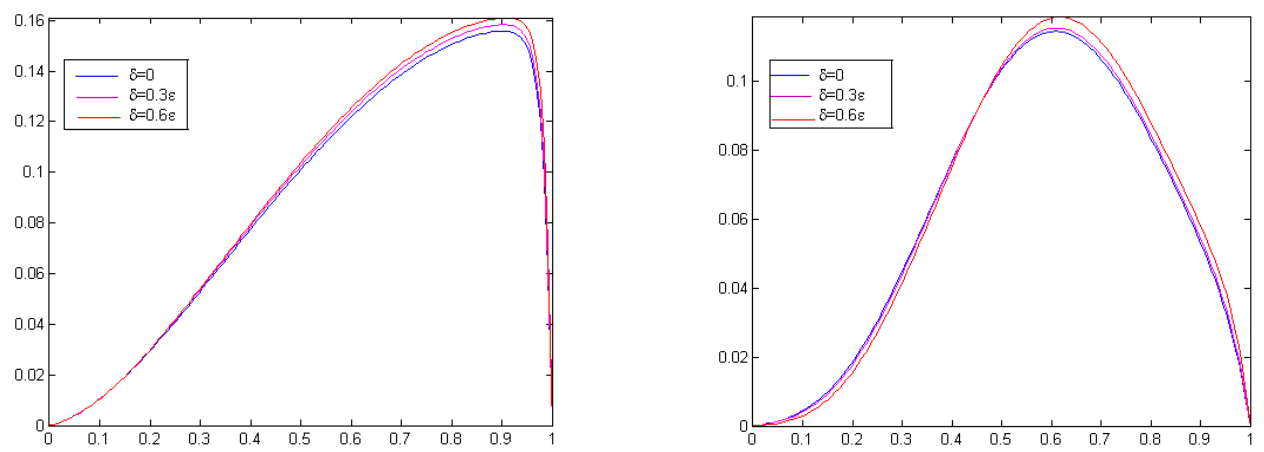

(a)

Figure 3: Effect of the delay on solution on (a) Example 1, on (b) Example 2, for $\varepsilon=2^{-2}$. (For interpretation of the references to color in this figure legend, the reader is referred to the web version of this article, DOI:10.5556/j.tkjm.53.2022.3638)
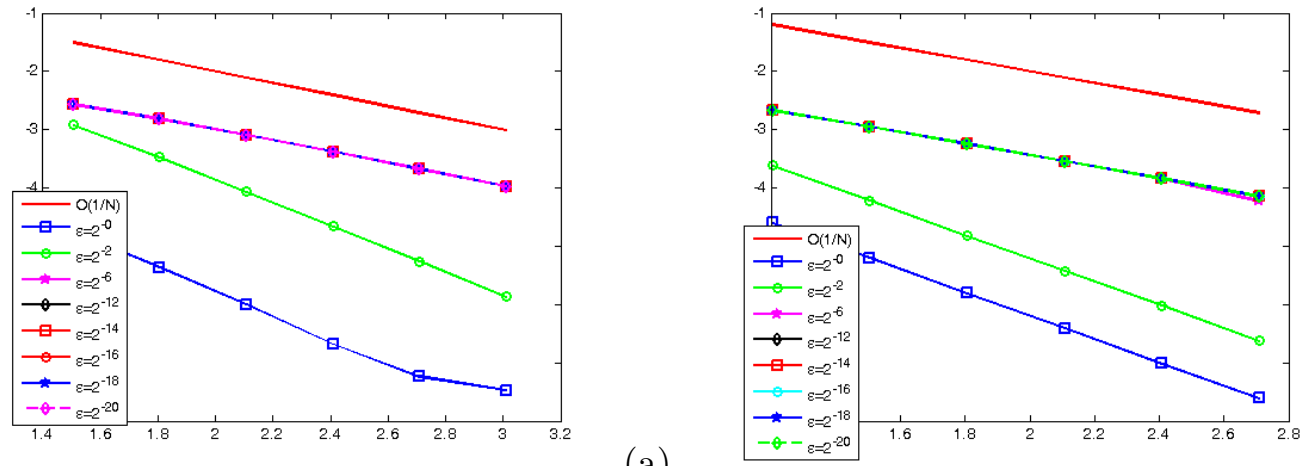

(a)

Figure 4: The Log-Log plot of the maximum absolute error on (a) Example 1, on (b) Example 2, for different values of $\varepsilon$. (For interpretation of the references to color in this figure legend, the reader is referred to the web version of this article, DOI:10.5556/j.tkjm.53.2022.3638)

\section{Acknowledgments}

The authors thank the referees for their careful reading and their fruitful comments on the manuscript.

\section{References}

[1] K. Bansal, K. K. Sharma, $\varepsilon$-Uniform numerical technique for the class of time dependent singularly perturbed parabolic problems with state dependent retarded argument arising from generalised Stein's model of neuronal variability, Differ. Equ. Dyn. Syst., (2016), 1-28.

[2] K. Bansal, K. K. Sharma, Parameter uniform numerical scheme for time dependent singu- 
larly perturbed convection-diffusion-reaction problems with general shift arguments, Numer. Algorithms, 75:1(2017), 113-145.

[3] K. Bansal, P. Rai, K. K. Sharma, Numerical treatment for the class of time dependent singularly perturbed parabolic problems with general shift arguments, Differ. Equ. Dyn. Syst., 25:2(2017), 327-346.

[4] C. Clavero, J. L. Gracia and J. C. Jorge, High-order numerical methods for one-dimensional parabolic singularly perturbed problems with regular layers, Numer. Methods Partial Differential Equations, 21:1 (2005), 149-169.

[5] I. T. Daba and G. F. Duressa, Extended cubic B-spline collocation method for singularly perturbed parabolic differential-difference equation arising in computational neuroscience, International Journal for Numerical Methods in Biomedical Engineering, 37:2 (2021), e3418.

[6] V. Gupta, M. Kumar and S. Kumar, Higher order numerical approximation for time dependent singularly perturbed differential-difference convection-diffusion equations, Numer. Methods Partial Differential Equations, 34(2018), 357-380.

[7] T. Kellogg and A. Tsan, Analysis of some difference approximations for a singular perturbation problem without turning point, Math. Comput., 32(1978), 1025-1039.

[8] D. Kumar and M. K. Kadalbajoo, A parameter-uniform numerical method for timedependent singularly perturbed differential-difference equations, Appl. Math. Model., 35:6(2011), 2805-2819.

[9] C. G. Lange and R. M. Miura, Singular perturbation analysis of boundary value problems for differential-difference equations III. Turning point problems, SIAM J. Appl. Math., 45:5(1985), 708-734.

[10] C. G. Lange and R. M. Miura, Singular perturbation analysis of boundary value problems for differential-difference equations. V. Small shifts with layer behavior, SIAM J. Appl. Math., 54:1(1994), 249-272.

[11] J. D. Murray, Mathematical Biology, Springer, Berlin, 1993

[12] J. D. Murray, Mathematical Biology I: An Introduction, Springer, New York, 2002

[13] M. Musila, P. Lánskỳ, Generalized Stein's model for anatomically complex neurons, BioSystems, 25:3(1991), 179-191.

[14] R. E. O'Malley, Singular perturbation methods for ordinary differential equations, Springer, 1991.

[15] V. P. Ramesh and M. K. Kadalbajoo, Upwind and midpoint upwind difference methods for time-dependent differential difference equations with layer behavior, Appl. Math. Comput., 202:2(2008), 453-471.

[16] R. N. Rao and P. P. Chakravarthy, Fitted Numerical Methods for Singularly Perturbed 1D Parabolic Partial Differential Equations with Small Shifts Arising in the Modeling of Neuronal Variability, Differ. Equ. Dyn. Syst., (2017), 1-18.

[17] H. G. Roos, M. Stynes and L. R. Tobiska, Numerical methods for singularly perturbed differential equations, Springer Science \& Business Media, 2008. 
[18] M. Shivhare, P. C. Podila, H. Ramos and J. Vigo-Aguiar, Quadratic B-spline collocation method for time dependent singularly perturbed differential-difference equation arising in the modeling of neuronal activity, Numer. Methods Partial Differential Equations, (2021), In press.

[19] R. B. Stein, A theoretical analysis of neuronal variability, Biophys. J., 5:2(1965), 173-194.

[20] R. B. Stein, Some models of neuronal variability, Biophys. J., 7:1(1967), 37-68.

[21] H. Tian, The exponential asymptotic stability of singularly perturbed delay differential equations with a bounded lag, J. Math. Anal. Appl., 270:1(2002), 143-149.

[22] M. M. Woldaregay and G. F. Duressa, Uniformly convergent numerical method for singularly perturbed delay parabolic differential equations arising in computational neuroscience, Kragujevac J. Math., 46:1(2022), 65-84. In Press

[23] M. M. Woldaregay and G. F. Duressa, Parameter uniform numerical method for singularly perturbed parabolic differential difference equations, J. Nigerian Math. Soc., 38:2(2019), 223-245.

[24] M. M. Woldaregay, W. T. Aniley and G. F. Duressa, Novel Numerical Scheme for Singularly Perturbed Time Delay Convection-Diffusion Equation, Advances in Mathematical Physics, 2021(2021), 1-13.

Mesfin Mekuria Woldaregay Department of Mathematics, Jimma University, Jimma, Ethiopia

E-mail: msfnmkr02@gmail.com

Gemechis File Duressa Department of Mathematics, Jimma University, Jimma, Ethiopia

E-mail: gammeef@gmail.com 